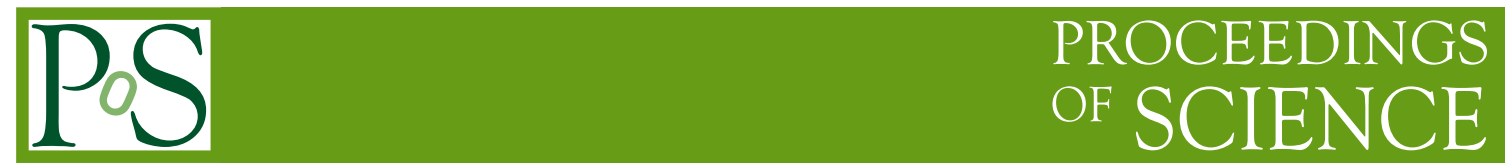

\title{
A Cosmic Vision Beyond Einstein
}

\section{Eric V. Linder*}

Berkeley Lab \& University of California, Berkeley USA

The acceleration of the cosmic expansion is a fundamental challenge to standard models of particle physics and cosmology. The new physics of dark energy may lie in the nature of gravity, the quantum vacuum, or extra dimensions. I give a brief overview of the puzzles and possibilities of dark energy, and discuss the confrontation of a wide variety of "beyond Einstein" models with the latest data, showing what we currently know and what we must seek to learn. Next generation experiments using a variety of cosmological probes will deeply explore dark energy, dark matter, and gravitation.

Identification of Dark Matter 2008

Stockholm, Sweden

August 18-22, 2008

${ }^{*}$ Speaker. 


\section{Introduction}

What is the universe made of and how does it work? These are the very basic, very fundamental questions displaying our current ignorance of $95 \%$ of the total energy in the universe. But that we publicly ask them also indicates that we have hopes, and challenging plans, for how to answer them. These questions and plans lie at the heart of the European Space Agency's Cosmic Vision program and the Joint Dark Energy Mission of NASA's Beyond Einstein program and the US Department of Energy.

With the discovery of the accelerating expansion of the universe we clearly realize that the Standard Model of the particle physics of baryons, photons, etc., augmented by dark matter, is insufficient to explain the majority of the cosmic energy density. The acceleration, or gravitational repulsion, property implies components beyond the standard ones dominated by rest mass or relativistic energy, to suggestions of quantum fluctuations of the vacuum, new high energy physics fields, or extensions of general relativity. To see which way the physics lies, we indeed need a cosmic vision beyond Einstein.

In §2 I present an overview of some of the key theoretical questions and the cosmological observational tools we have and can further develop to answer them. Using the latest sets of data, $\$ 3$ examines whether we have already narrowed down to a model essentially that of the static dark energy of Einstein's cosmological constant or whether there is still ample room beyond Einstein. In $\S 4$, methods for making our cosmic vision sharper, clearer, and steadier with space experiments are discussed.

\section{Ideas about the Accelerating Universe}

From the Equivalence Principle, we are used to thinking about the energy-momentum contents of the universe as inducing curvature in spacetime, the simplest picture being a ball of mass-energy bending the rubber sheet of spacetime such that a marble (test particle) bends its path toward it, experiencing an attraction we interpret as gravitation. However, if the pressure contribution to the energy-momentum of a component is sufficiently negative, then the overall gravitational mass of the ball of "negative pressure stuff" - given the more euphonious name of quintessence - is itself negative ${ }^{1}$. The rubber sheet potential well becomes a hill, and the marble retreats from the ball, experiencing repulsion. This is one explanation of the cause of acceleration in the cosmic expansion: rather than the gravity of the average contents of the universe pulling things together and slowing the expansion, it pulls them apart, speeding up the cosmic expansion.

The outstanding surprise about such repulsion is that it is not a highly exotic phenomenon witnessed only in extreme conditions in some small corner of the universe, but that it dominates our current universe, with over $70 \%$ of the contents acting in such a mysterious manner. One possibility for this physics beyond the standard model is Einstein's cosmological constant, an eternally unchanging pressure, negatively equal to its energy density, uniform in space and time. Such constancy raises the question of why it comes to dominate the universe now, given our current picture

\footnotetext{
${ }^{1}$ An alternative, not widely accepted [Van Acolyen(2008), Rosenthal \& Flanagan(2008)] idea for acceleration involves backreaction of nonlinear structure on the expansion. This effectively seeks to use strongly positive pressure to density ratio (equation of state) but negative energy density.
} 
of a universe of matter structures co-existing with acceleration: a factor four in the expansion factor into the past we would never have noticed observationally the cosmological constant, while a factor four in the future arrays of clusters of galaxies would be rare things to our telescopes. See [Frieman, Turner, \& Huterer(2008)] for further discussion.

Beyond the cosmological constant there are vast fields of dynamical (time varying) dark energy explanations, such as quintessence. This dichotomy between static and dynamic is a fundamental issue to resolve. We need to know not just how much dark energy there is (its fractional contribution $\Omega_{\mathrm{de}}$ to the total energy density), but how springy/stretchy it is to the spacetime. The ratio of the pressure to the energy density, called the equation of state $w$, is a (time dependent) measure of this. By measuring through cosmological observations some instantaneous value $w$ and some measure of time variation $w^{\prime}=d w / d \ln a$, where $a$ is the expansion factor (redshift $z=a^{-1}-1$ ), we can not only potentially exclude the cosmological constant $\left(w=-1, w^{\prime}=0\right)$ explanation, but actually be guided to a class of physics responsible for the explanation.

[Caldwell \& Linder(2005)] showed the utility of the $w-w^{\prime}$ phase space to subdivide behaviors into thawing models (departing from a cosmological constant-like static behavior in the past) and freezing models (approaching cosmological constant behavior in the future). Just recently, [de Putter \& Linder(2008)] demonstrated that proper choice of the particular measures of $w$ and $w^{\prime}$ could highlight the differences in physics and separate dark energy models into distinct families (see Fig. 1). Moreover, using these variables $w_{0}=w(a=1)$, the value today, and for the time variation $w_{a}=-d w / d a(a=0.8)$, traces the observables of distances $d(z)$ and Hubble expansion rate $H(z)$ to $10^{-3}$ level accuracy - better than needed for robust interpretation of next generation experiments. Thus, a simple model-independent theoretical framework is in place for understanding what observations tell us about the accelerating universe.

To carry out the mapping of the universe, we have several, complementary probes. The cosmic microwave background radiation (CMB) is a direct probe of the early universe, and is highly useful for breaking degeneracies between dark energy and other parameters. Three-dimensional surveys of galaxies and clusters of galaxies can study both expansion and the growth of structure, e.g. through the matter power spectrum and its baryon acoustic oscillation (BAO) features, and through weak gravitational lensing (WL). Supernovae give a direct probe of the cosmic expansion over the entire acceleration epoch and back into the decelerating epoch. These techniques have important complementarities, with the CMB and BAO data anchored in the high redshift, matter dominated era, WL data most sensitive at intermediate redshifts, and supernovae anchored in the current, accelerating era. Together they give important crosschecks, systematics tests, and tight constraints on dark energy properties.

\section{Are We Done?}

With such cosmological tools, it is not surprising there is a great deal of activity. Unlike weather, where everybody talks about it but nobody does anything about it, with dark energy everybody wants to have something to do with it. Most recently, the greatest observational advances have come from the Type Ia supernova technique. [Kowalski et al.(2008)] carried out a complete reanalysis of all published data sets, amounting to 307 supernovae after quality cuts, and applied robust, blind statistical and cosmological analysis. Systematic uncertainties now contribute equally 

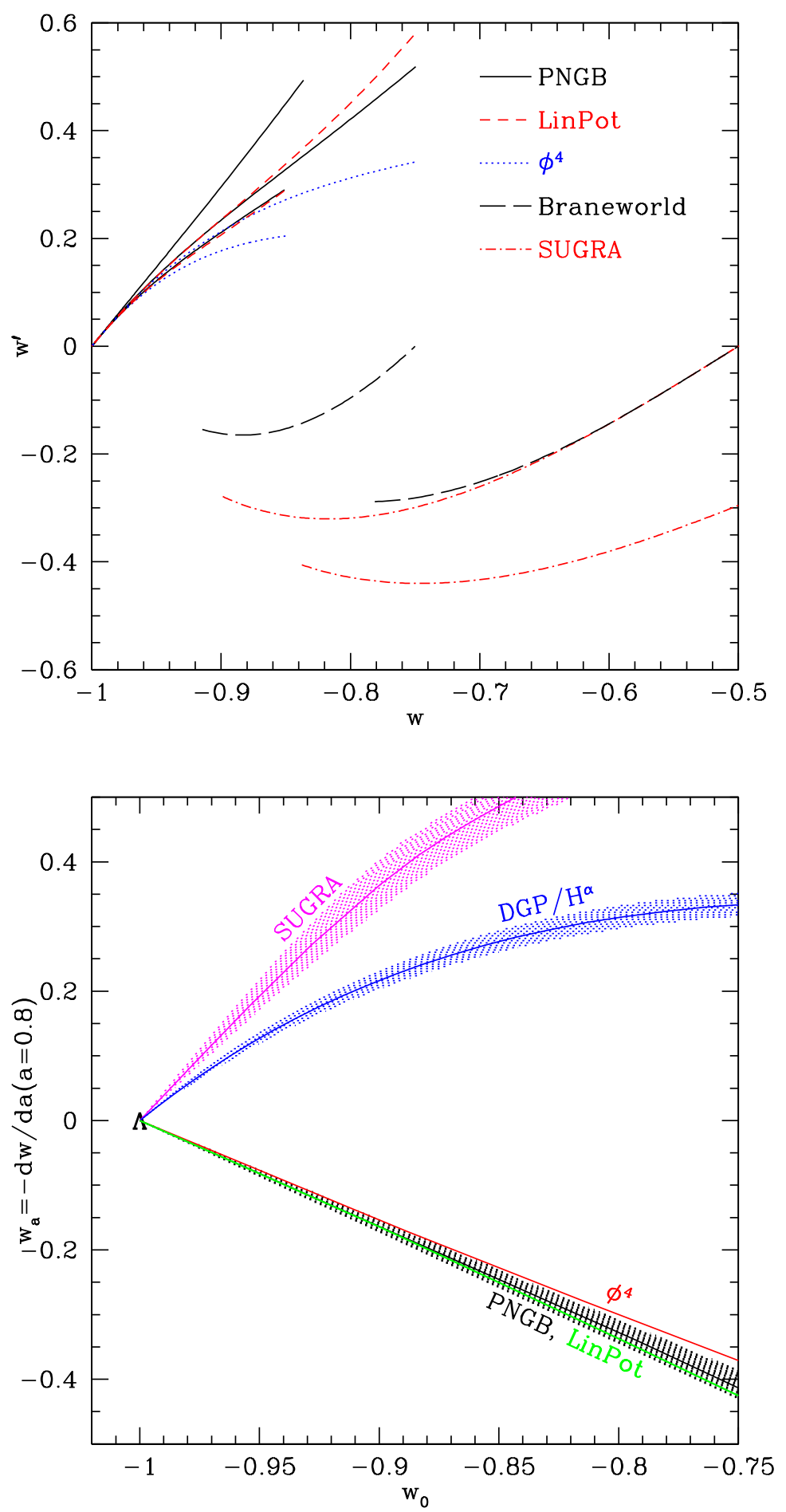

Figure 1: The dynamics of dark energy models in the $w-w^{\prime}$ phase space is diverse between models, and within models as the parameters of the potential are varied, although still separated into thawing (upper) and freezing (lower) regions [top panel]. In terms of the calibrated dark energy parameters $w_{0}$ and $w_{a}$, however [bottom panel; note vertical axis is flipped], models and families lie in tightly homogeneous regions, even scanning over the parameters within the potentials. From [de Putter \& Linder(2008)]. 
with statistical precision. They find no significant deviations in the mean distance-redshift relation among the 13 data sets, no truly significant deviations in the redshift dependence or slope of the residuals from the mean, and no evidence of uncorrected evolution through tests of redshift and population subsamples. Thus the cosmology results appear robust at the current level.

Fitting for a constant $w$ equation of state by combining SN, BAO, and CMB data, [Kowalski et al.(2008)] finds $w=-0.969 \pm 0.061$ (stat) \pm 0.065 (sys). This may lead some people to ask "are we done?", i.e. since $w$ is consistent with the cosmological constant value of $w=-1$, should we be content with that as the answer? Emphatically not. We do not know that $w(z)=-1$, allowing for time variation, or even anything at all about dark energy properties at $z>1$. [Kowalski et al.(2008)] find constraints on the value of $w$ range from -6 to +1 at $68 \% \mathrm{cl}$ in a redshift bin from $z=1-2$.

While investigating redshift-bin values of $w$ or parametrizations gives great model-independent freedom, we can also compare specific physical models to the cosmological constant $\Lambda$ and ask whether the data prefer the cosmological constant. This was done for 10 reasonably physically motivated models in [Rubin et al.(2008)], ranging over thawing (e.g. see Fig. 2) and freezing scalar fields, phase transitions, extended gravity, and geometric dark energy. The results were that essentially all the models survive, with a couple in fact being modestly preferred over the cosmological constant.

The overriding conclusion is that essentially all types of physics are still in play, and that improvements in systematics control will have a major impact. For example, the extra dimensional braneworld cosmology has a penalty of only +2.7 in $\Delta \chi^{2}$ relative to $\Lambda \mathrm{CDM}$, but this would increase to a highly significant +15 if the systematics uncertainty were removed. A homogeneous data set of supernovae and a uniform analysis pipeline are key to revealing the physics, as will be the future sensitivity to the time variation $w(z)$. So to truly advance our knowledge of the nature of dark energy we must obtain better data, with next generation experiments.

\section{Clear Vision}

To obtain the redshift range needed for sensitivity to the time variation $w(z)$ and to improve systematics control for the supernova and weak lensing methods, observations are driven to space. Figure 3 shows how the innate cosmology dependence and degeneracies between parameters requires high redshift observations.

Space observations have strong advantages for both supernova and weak lensing probes. For supernovae, the extended wavelength range from the optical into the near infrared allowed by being above the atmosphere gives a greater lever arm on dust extinction systematics, and the near infrared enables greater redshift depth, increasing cosmology sensitivity. Weak lensing uses the apparent shape distortions of distant galaxies by intervening mass concentrations to probe combinations of distances and growth of structure. The noise in these subtle (percent level) distortions is much less from the stable space environment than at any ground based telescope, which must contend with weather, gravity, and the atmosphere [Kasliwal et al.(2008)], and the signal is clearer due to the high resolution of space observations and the stable point spread function [Massey, Refregier, \& Rhodes(2004)].

Next generation space experiments such as from the Cosmic Vision program or Joint Dark Energy Mission will enable deep surveys mapping the last 10 billion years of cosmic expansion 


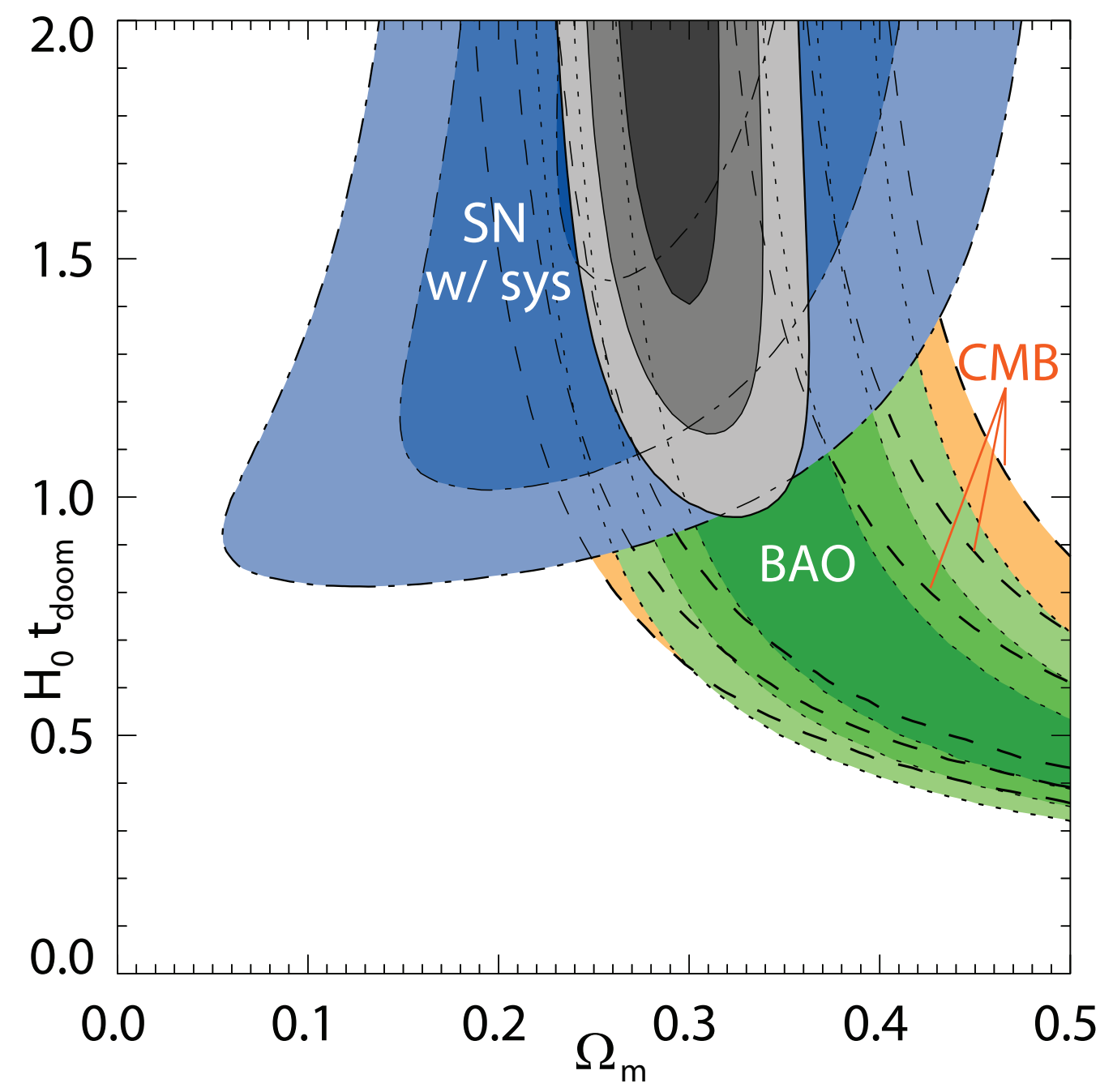

Figure 2: The future expansion history in the linear potential model has a collapse, or cosmic doomsday, at a finite time in the future. While $t_{\text {doom }}=\infty$ corresponds to the $\Lambda$ CDM model, current data impose only $t_{\text {doom }}>1.24 H_{0}^{-1}$ from now at $95 \%$ confidence. From [Rubin et al.(2008)].

over 10000 times the area of the Hubble Space Telescope Deep Field (HDF), and wide surveys of cosmic structure over 10 million times the area of HDF, nearly as deep, and covering optical through near infrared wavelengths. Widefield space surveys also maximize discovery potential, capable of mapping the dark matter skeleton of the universe over areas 5000 times that of the HST Cosmos survey [Massey et al.(2007)], probing inflationary parameters and non-Gaussianity through precision measurements of the matter power spectrum, and assembling an impressive 40 trillion color-resolution elements on the sky -20 times that planned from next generation ground surveys. 


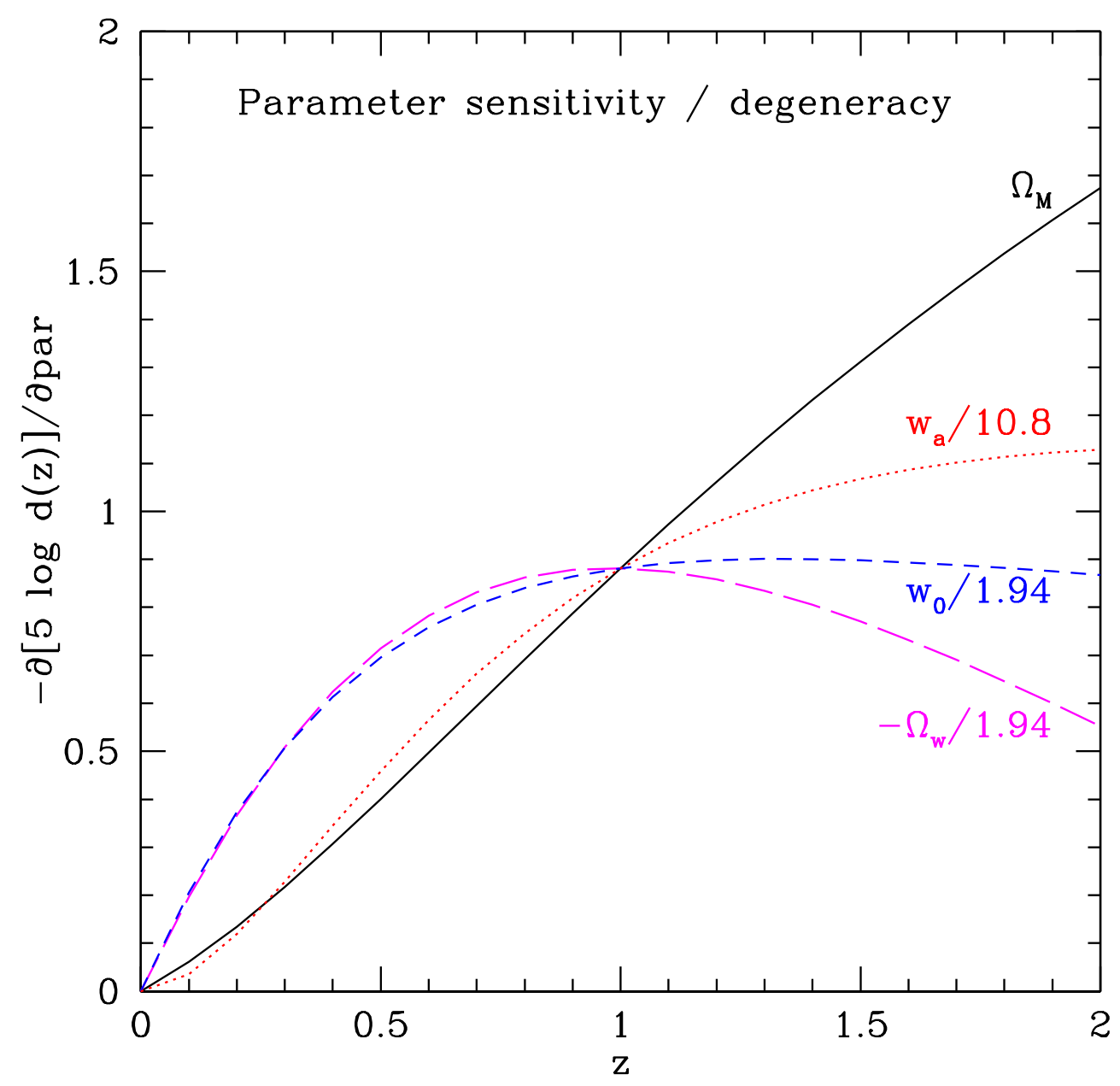

Figure 3: Cosmological parameter sensitivities for the magnitude-redshift relation, $5 \log d(z)$, are plotted for the matter density $\Omega_{m}$, dark energy density $\Omega_{w}$ (spatial curvature is allowed), and dark energy equation of state parameters $w_{0}$ and $w_{a}$. Since only the shapes of the curves matter for degeneracy, parameters are normalized to make this more evident. Observations out to $z \geq 1.5$ are required to break the degeneracies. From [Linder(2008)].

\section{Summary}

Cosmic acceleration is a fundamental mystery demonstrating that our understanding of the universe and its contents is woefully incomplete. Indeed it puts us on the brink of a revolution in physics, though whether in quantum fields, gravitation, or cosmology is unknown. Great efforts are being made to explore this, but current data do not tell us that the cosmological constant $\Lambda$ is the answer. Indeed, two strikes against it are that Einstein and everyone else have failed for 90 years to explain the 120 orders of magnitude discrepancy from expectations, and that the one prior period of acceleration we know of - inflation - ended, and so clearly had time variation and dynamics.

The need to understand the dynamics of the dark energy responsible for the current acceleration is crucial, and this requires going well beyond current data. Fortunately, clear ideas exist 
of how to use several different, complementary probes in admittedly challenging, robust measurements. With high resolution and low systematics, and multiple complementary probes of expansion and growth, we can robustly explore the next physics, testing cosmology, general relativity, quantum vacua, and dark matter. Next generation space experiments will make sharper, clearer, and steadier our cosmic vision beyond Einstein.

\section{Acknowledgments}

I am grateful to Joakim Edsjö and the organizers of IDM2008 for the invitation and wonderful hospitality. This work has been supported in part by the Director, Office of Science, Office of High Energy Physics, of the U.S. Department of Energy under Contract No. DE-AC02-05CH11231.

\section{References}

[Van Acolyen(2008)] K. Van Acolyen, LTB Solutions in Newtonian Gauge: from Strong to Weak Fields, [arXiv:0808.3554]

[Rosenthal \& Flanagan(2008)] E. Rosenthal \& E.E. Flanagan, Cosmological Backreaction and Spatially Averaged Spatial Curvature, [arXiv:0809.2107]

[Frieman, Turner, \& Huterer(2008)] J. Frieman, M. Turner, \& D. Huterer, Dark Energy and the Accelerating Universe, Annual Reviews of Astronomy and Astrophysics in press [arXiv:0803.0982]

[Caldwell \& Linder(2005)] R.R. Caldwell \& E.V. Linder, Limits of Quintessence, Phys. Rev. Lett. 95 141301 (2005) [arXiv:astro-ph/0505494]

[de Putter \& Linder(2008)] R. de Putter \& E.V. Linder, Calibrating Dark Energy, JCAP in press [arXiv:0808.0189]

[Kowalski et al.(2008)] M. Kowalski et al., Improved Cosmological Constraints from New, Old, and Combined Supernova Datasets, ApJ 686749 (2008) [arXiv: 0804 .4142]

[Rubin et al.(2008)] D. Rubin et al., Looking Beyond Lambda with the Union Supernova Compilation, ApJ submitted [arXiv:0807.1108]

[Linder(2008)] E.V. Linder, Mapping the Cosmological Expansion, Reports on Progress in Physics 71 0560901 (2008) [arXiv:0801.2968]

[Kasliwal et al.(2008)] M.M. Kasliwal, R. Massey, R.S. Ellis, S. Miyazaki, J. Rhodes, Comparison of Weak Lensing Measurements from Ground-and Space-Based Facilities, ApJ 68434 (2008) [arXiv:0710.3588]

[Massey, Refregier, \& Rhodes(2004)] R. Massey, A. Refregier, \& J. Rhodes, Probing Dark Matter and Dark Energy with Space-Based Weak Lensing, [arXiv: astro-ph/0403229]

[Massey et al.(2007)] R. Massey et al., Dark Matter Maps Reveal Cosmic Scaffolding, Nature 445286 (2007) [arXiv:astro-ph/0701594] 